
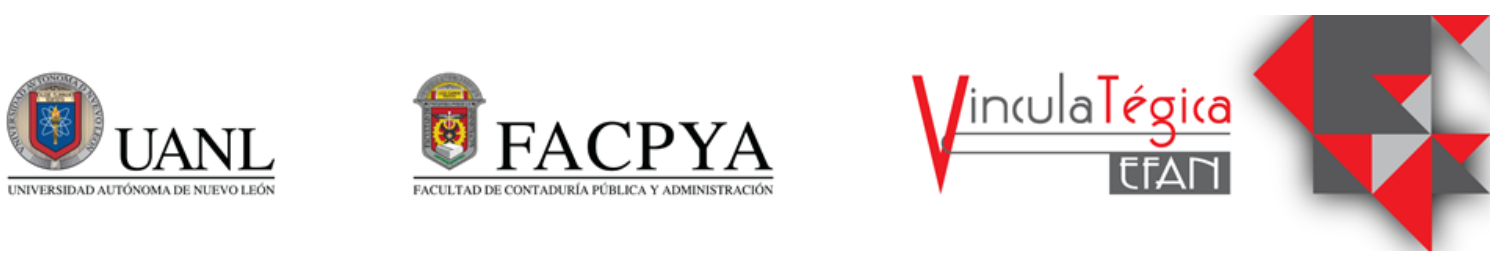

\title{
Factores que influyen en las MiPyMES en tiempos de Covid-19
}

\author{
Allen Ricardo Torres Garza ${ }^{1}$, María Margarita Carrera Sánchez y Abel Partida Puente ${ }^{3}$ \\ ${ }^{1}$ Universidad Autónoma de Nuevo León, Facultad de Contaduría Pública y Administración, Centro de \\ Desarrollo Empresarial y Posgrado,allen_torres@hotmail.com, Av.Universidad S/N, Col. Ciudad \\ Universitaria, C.P. 66455, San Nicolás de los Garza, Nuevo León, México, 8183294080 \\ ${ }^{2}$ Universidad Autónoma de Nuevo León, Facultad de Contaduría Pública y Administración, Centro de \\ Desarrollo Empresarial y Posgrado, magaly_carrera@hotmail.com, Av. Universidad S/N, Col. Ciudad \\ Universitaria, C.P. 66455, San Nicolás de los Garza, Nuevo León, México, 8183294080 \\ ${ }^{3}$ Universidad Autónoma de Nuevo León, Facultad de Contaduría Pública y Administración, Centro de \\ Desarrollo Empresarial y Posgrado, abelpartida@hotmail.com, Av. Universidad S/N, Col. Ciudad \\ Universitaria, C.P. 66455, San Nicolás de los Garza, Nuevo León, México, 8183294080
}

Información del artículo revisado por pares

Fecha de aceptación: junio-2021

Fecha de publicación en línea: diciembre-2021

DOI: https://doi.org/10.29105/vtga7.1-87

\section{Resumen}

El impacto social, económico y productivo que ha tenido el Covid-19, indudablemente será material de estudio durante años, el mundo se encuentra aún inmerso entre el primer brote y un rebrote. Con la llegada del Covid -19 se han generado grandes cambios sociales y económicos, las empresas se han visto obligadas a modificar sus modelos de negocio para mantenerse vigentes durante la presente contingencia sanitaria. El objetivo del presente trabajo de investigación tiene como finalidad describir los factores que influyen en las Mipymes y sus efectos en estas. El análisis realizado se definió con base a un marco de referencia, técnica utilizada documental y bibliográfica, que permiten identificar los factores que se presentan con regularidad en tiempos de pandemia. Los resultados del presente estudio demuestran que, al implementar cambios tecnológicos, entornos colaborativos, innovaciones y nuevos modelos de negocio, influyen positivamente en las Mipymes en tiempos de pandemia. En el
The social, economic, and productive impact that Covid-19 has had undoubtedly be study for many years, the world is still immersed between the first outbreak and a regrowth. With the arrival of Covid -19 , great social and economic changes have been generated, companies have been forced to modify their business models to stay current during the health contingency. The aim of this research work is to describe the factors that influence MSMEs and their effects on them. The analysis carried out was defined based on a reference framework, a documentary and bibliographic technique used, which allow identifying the factors that occur regularly in times of pandemic. The results of this study show that, by implementing technological changes, collaborative environments, innovations and new business models, influence MSMEs positively in times of pandemic. In the present work, it is concluded that empirical research indicates that the study variables have served as 
presente trabajo se concluye que las investigaciones empíricas señalan que las variables de estudio han servido de apoyo para que las Mipymes puedan mantenerse y no desaparezcan ante la contingencia sanitaria originada por el Covid-19.

Palabras clave: Covid-19, Entornos Colaborativos, Innovación, MiPyMES, Modelo de Negocio.

\section{INTRODUCCIÓN}

En el mes de diciembre del 2019, en Wuhan, China Central, se descubrió un nuevo coronavirus el cual es causante de un síndrome respiratorio agudo severo el SARS-CoV-2, comúnmente llamado Covid-19, como lo llamaremos de ahora en delante (Sharma et ál., 2020).

Actualmente existe mucha incertidumbre en relación con la capacidad de los consumidores para adaptarse a estos nuevos tiempos, principalmente por el tema de hacer frente a la contingencia sanitaria ya que esta origina cambios en los mercados económicos y en la conducta de la sociedad (Campbell et ál., 2020). Es importante comentar que de la actual crisis sanitaria nacerán nuevos hábitos o conductas por parte de los consumidores, entre estos podremos encontrar un mayor uso de nuevas tecnologías para la adquisición de productos y servicios, aunado a formas innovadoras para temas recreativos y educativos (Sheth, 2020).

Las micro, pequeña y mediana empresa (Mipymes), se han visto afectadas fuertemente, necesitan modificar y replantear sus estrategias financieras, transformar $y$ adaptar decisiones institucionales, incluir canales digitales en sus procesos y aprovechar al máximo las oportunidades que se están presentando en temas de innovación tecnológica (Dannenberg et al., 2020).

Conforme avanza la pandemia las empresas han realizado cambios en sus actividades y modelos de negocio, esto en temas de generación y creación de valor para sus clientes, principalmente en temas de tiempos de respuesta y disminución de costos, todo esto previa identificación de factores support so that MSMEs can be maintained and not disappear in this health contingency caused by Covid-19.

Keywords: Business Model, Collaborative Environments, Covid-19, Innovation, MSME's. JEL: M10, M19, O32.

clave que generen nuevas oportunidades de desarrollo para la empresa (Gamero y Ostos, 2020).

En Latinoamérica y el Caribe, el ambiente social es muy complejo ya que se calcula que 2.7 millones de empresas, el 19\% de la región han cerrado sus operaciones. El mayor número de empresas afectadas son las Mipymes. Esto significa la pérdida de trabajo de más de 8.5 millones de individuos, que representan una quinta parte del total de la zona (CEPAL, 2020).

El objetivo del presente trabajo de investigación tiene como finalidad describir los factores que influyen en las Mipymes y sus efectos en estas. El análisis realizado se definió con base a un marco de referencia que permite identificar los factores que se presentan con regularidad en tiempos de pandemia, los problemas que han enfrentado y las modificaciones que han tenido que realizar en temas operacionales enfocándonos principalmente en los cambios tecnológicos, entornos colaborativos, innovaciones y los modelos de negocio.

El método utilizado en el presente estudio es de tipo documental ya que se realizaron consultas en diferentes instrumentos como revistas, investigaciones empíricas, registros y diferentes fuentes existentes y bibliográficas ya que se revisó material bibliográfico existente respecto a nuestro tema de investigación.

En México, se estima que el $96 \%$ de los afectados serán las Mipymes y el $58 \%$ podrían desaparecer, ya que la tercera parte de ellas ya han reportado despidos y cierres parciales (El Heraldo de México, 2020). De acuerdo con este planteamiento del problema 
que afecta a las empresas se lleva a cabo el siguiente cuestionamiento ¿Cuáles son los factores que influyen en las Mipymes en tiempos de pandemia?

La investigación actual está dividida en cuatro secciones, en la primera encontramos algunas reflexiones relacionadas con las Mipymes en tiempos del Covid-19 y la manera en la que estas se han adaptado. En la segunda visualizaremos un análisis teórico de las variables de estudio junto con investigaciones empíricas relacionadas, estos estudios relacionados con las Mipymes y el COVID-19, en la tercera sección encontraremos los principales resultados de las investigaciones analizadas y por último se realizan las principales conclusiones y reflexiones de acuerdo con la revisión de la literatura que buscan ser un punto de partida para la continuación de investigaciones futuras relacionadas con las variables de estudio.

\section{MARCO TEÓRICO}

Para identificar los avances relacionados con el tema de investigación, se realizó una revisión literaria de artículos científicos de tipos teóricos, conceptuales y empíricos, la búsqueda se realizó principalmente en las siguientes bases de datos, Dialnet, ScienceDirect, ProQuest, Scopus, Scielo, Redalyc y Google Académico.

\subsection{Las Mipymes en tiempos del Covid-19.}

Latinoamérica es un territorio cada vez de mayor interés en asuntos relacionados con las Mipymes ya que están son un gran generador de empleo en la región. Una de las causas por las que hoy por hoy existe interés en el estudio de estas empresas es que en la mayor parte de los países encontramos una cantidad importante de Mipymes, las cuales favorecen a las economías de los países en donde se encuentran ubicadas (Chiao, Yang y $\mathrm{Yu}, 2006)$.

Existe gran cantidad de causas por las que las Mipymes y particularmente en economías emergentes, han despertado el interés del mundo, entre las principales razones encontramos, el gran número de empleos que estas generan, son puntos importantes de innovación, la interacción con estas es muy fluida, la rápida toma de decisiones y su cercanía con los clientes, son algunas de las ventajas que este tipo de empresas tienen (Katua, 2014).

Para Segura (2020), "A nivel global, las empresas y las personas están entrando en una etapa de menor consumo, menor demanda de bienes y servicios y, por lo tanto, menor interacción económica en los mercados".

Por otro lado, para américa latina, es importante mencionar temas como la ciencia, la innovación y el desarrollo productivo para hacer frente al Covid-19, adaptando estos temas a los entornos empresariales y de gestión pública, existen muchas necesidades y oportunidades que se deben de aprovechar en estos tiempos incluida la capacidad de reacción que tengan las diferentes economías de la región (BID, 2020).

\subsection{Cambios tecnológicos, una visión teórica.}

En relación con las dimensiones tanto empresariales como organizativas de las TIC, identificamos gran cantidad de recursos que ayudan a manipular información y que llevan al desarrollo y crecimiento económico de cualquier empresa, esto gracias a que las TIC provén a las organizaciones grandes oportunidades de administrar el conocimiento existente, aumentan su flexibilidad, adicionalmente incrementan la capacidad de desarrollo de la misma en beneficio de su crecimiento y la ayuda en potenciar su independencia (Thompson y Strickland, 2004).

Las Tecnologías de Información y Comunicación (TIC) forman un campo complejo en donde se pueden encontrar gran cantidad de definiciones y clasificaciones, estas se componen principalmente por recursos como hardware, software y una gran cantidad de dispositivos electrónicos entrelazados unos con otros, con capacidad de formar una red universal de herramientas para la acumulación de datos con la finalidad de crear información (Cobo-Romani, 2009).

Según Gálvez et al. (2014), las tecnologías de información y comunicación son los medios electrónicos de almacenamiento, captura, enlace y 
transmisión de datos e información para facilitar el diseño de estrategias que fomentaran el desarrollo de la empresa, influyen de forma positiva rentabilizando a las organizaciones que las usan de manera efectiva.

La administración de la tecnología es uno de los temas que en las anteriores tres décadas ha sido objeto de estudio, todo esto originado por la correcta administración de los métodos y recursos tecnológicos utilizados de manera eficiente por las empresas, es imperativo mencionar que se ha logrado ser cada vez más eficiente en procesos internos y esto por consecuencia a detonado en que los sectores económicos sean cada vez más competitivos, incluyendo las Mipymes (Medellín, 2010; Duque y Garzón, 2015).

La digitalización no solo da la oportunidad a las Mipymes de adaptarse a una crisis como la actual, sino que otorga un apoyo considerable para promover la sostenibilidad dentro de las empresas. El uso adecuado de las nuevas tecnologías y la digitalización ayudan a las empresas a incrementar el desarrollo sostenible disminuyendo los traslados de la fuerza laboral, promoviendo la flexibilidad y conciliación laboral e impulsa una administración más sostenible de la cadena de suministro organizacional (PwC, 2019).

De acuerdo con Akpan et ál. (2020) existen dificultades para que las Mipymes se adapten a los nuevos escenarios relacionados con la transformación tecnológica ya que la cultura que impera en estas es muy conservadora, adicionalmente menciona que existen importantes ventajas en la implementación de nuevas tecnologías.

Los procesos de transformación digital dependen en gran medida de la capacidad del recurso humano de la empresa, así como de la disposición que estos tengan para adaptarse a los cambios tecnológicos. Es importante mencionar que a medida que los cambios tecnológicos avanzan sus vulnerabilidades también aumentan y en este sentido es imperativo proteger los sistemas de actividad maliciosa o del uso indebido de estas tecnologías (Almeida y Monteiro, 2020).

Las micro, pequeñas y medianas empresas deben aprovechar todas las ventajas que les proporcionan los recursos tecnológicos y las redes sociales para difundir y promocionar sus bienes y servicios, uno de los mayores atrasos lo encontramos en los micro negocios, ya que solo el $17.2 \%$ usa internet y solo el $19.8 \%$ emplea una PC, esto aumenta en el sur del país, en donde encontramos que menos del $20 \%$ de las empresas usan internet, es importante mencionar que cuando se habla de ventas y compras por internet solo el $4.3 \%$ de las Mipymes realizan sus transacciones comerciales por este medio (INEGI, 2020).

\subsubsection{Cambios tecnológicos, estudios empíricos}

Tomando como referencia a Bermeo et ál. (2020) en su investigación con enfoque cuantitativo, con diseño no experimental, descriptivo y transversal donde analizaron el impacto generado a las pequeñas y medianas empresas con el uso de las TIC y herramientas tecnológicas, aplicando un cuestionario de 50 preguntas dicotómicas con elección de respuesta SI o NO, en escala de Likert aplicada a 150 Pymes, aplicada a los responsables de las diferentes áreas de la empresa encontramos que los elementos analizadas fueron el uso de las TIC, la utilización de herramientas tecnológicas y ventajas y desventajas con el uso de las TIC's, podemos encontrar como principal hallazgo que las TIC son utilizadas principalmente para temas de promoción y publicidad de los productos y servicios que ofrecen las empresas, adicionalmente encuentran un impacto positivo en temas de control, administración y seguridad de información al utilizar estas tecnologías de información y comunicación.

Heredia, Aguilar y Sainz (2020) realizaron un estudio de campo bajo un enfoque metodológico descriptivo de corte transversal para identificar con los gerentes de empresas cual ha sido su situación frente a la crisis del COVID-19 aplicando un cuestionario estandarizado con un coeficiente de confiabilidad alfa de Cronbach de 0.92 determinaron que en relación a las nuevas tecnologías estas son indispensables para enfrentar las condiciones cambiantes que se viven hoy en día ya que estas generan nuevas 
oportunidades de mercado y por consecuencia el desarrollo de innovaciones.

El trabajo presentado por Rodríguez et ál. (2020) nos da una visión de cambios tecnológicos relacionados e influenciados por el e-commerce en tiempos del Covid -19, su trabajo tiene un diseño de tipo documental, haciendo uso de métodos científicos teóricos y empíricos. La indagación de información se hizo a partir de fuentes primarias como artículos, informes técnicos y memorias de ponencias. El principal contenido es el de revelar como ha cambiado y evolucionado el uso del e-commerce en las Mipymes en tiempos de COVID-19. Como principales descubrimientos podemos mencionar la modificación en la forma de comercializar sus productos y servicios, ya que el comercio tradicional migro al comercio en línea, las Mipymes ahora consideran como un aliado a la transformación digita, el e-commerce es ahora un nuevo método de comercialización, que llegó para quedarse.

De acuerdo con Pérez, García y García (2021) en donde realizaron una investigación con un análisis exploratorio, mediante modelización de ecuaciones estructurales con mínimos cuadrados parciales (PLS-SEM), tomando datos de una encuesta aplicada a 76 directivos de empresas entre los meses de mayo y agosto 2020, esta encuesta fue desarrollada con preguntas abiertas sobre las distintas actividades aplicables a las nuevas tecnologías de información y comunicación cuantificado por una escala tipo Likert de 1 a 3, en donde encontramos Nivel 1 (Likert 1) infraestructura tecnológica básica, Nivel 2 (Likert 2) soluciones del ámbito general y Nivel 3 (Likert 3 ) industria 4.0 y servicios TIC avanzados. La hipótesis propuesta en esta investigación fue $\mathrm{H} 1$ : Existe una relación positiva y significativa entre el desarrollo de TIC de una empresa y su capacidad de adaptación ante el shock externo producido por la Covid-19, donde se concluyó que las tecnologías de información y comunicación como medida de implementación de cambio tecnológico permiten administrar de manera mes eficaz la información y la coordinación de actividades dentro de las empresas por lo que esto le permite adaptarse más rápido al nuevo entorno competitivo.

Uribe y Sabogal (2021) nos presentan en su investigación exploratoria descriptiva relacionada con cambios tecnológicos centrados en el marketing digital para las Mipymes por medio de un muestreo con representatividad estadística, aplicando encuestas y análisis de contenido un punto de vista enfocado en la teoría de que el marketing digital es realizado de manera informal por las empresas a lo que encontraron que las empresas tienen una gran área de oportunidad en temas de formalizar y profesionalizar sus áreas de marketing para la implementación de estrategias formales de marketing digital. Se debe de incluir presupuesto para temas como la capacitación y tecnología de nuevos desarrollos tecnológicos como el pago en línea, entre otros.

\subsection{Entornos colaborativos, una visión teórica.}

Los entornos colaborativos apoyan enormemente a las Mipymes a desarrollar nuevas formas de trabajo, así como nuevos productos y servicios (Gulati, 1998).

El entorno colaborativo se define como el proceso por el cual grupos de trabajo alcanzan objetivos en común, este proceso se desarrolla con herramientas tecnológicas llamadas actualmente tecnologías de información y comunicación con las cuales se facilita el trabajo y la gestión de la información en beneficio de la organización (Luna, 1999).

Otro punto importante es que este entorno también permite que las empresas puedan compartir experiencias y habilidades, todo esto tendrá en el futuro efectos positivos en el desempeño de las Mipymes (Argote e Ingram, 2000).

Es importante mencionar que el entorno colaborativo está calificado en la literatura como una estrategia empresarial que permite principalmente a las Mipymes cumplir varias actividades de innovación tal como lo realizan las grandes empresas (Narula, 2004).

La colaboración requiere que los individuos además de participar se relacionen para conseguir un determinado objetivo, en otras palabras, la colaboración involucra la participación "intencionada" y coordinada de 
los integrantes de un equipo de trabajo. Para incorporar a un sistema tecnológico la capacidad de colaboración es importante identificar a los integrantes del equipo que tienen las capacidades necesarias para desempeñar las tareas colaborativas (Sosa et ál., 2006).

Adicionalmente las Mipymes podrán aumentar su nivel de producción y desarrollo de la localidad y del país en donde estén instaladas (Biggs y Shah, 2006).

Los aprendizajes colaborativos han demostrado ser muy eficientes al ser desarrollados en ambientes virtuales donde estos se caracterizan por ser flexibles, adaptables y muy interactivos donde las diferentes audiencias o usuarios generalmente desarrollan proyectos y crean nuevas funciones para la organización (Marcano et ál., 2007).

$\begin{array}{crr}\text { Las relaciones } & \text { interpersonales y } \\ \text { profesionales } & \text { han } & \text { experimentado }\end{array}$ transformaciones significativas en tiempos del Covid-19. Durante el tiempo de cuarentena, las personas tuvieron que orientar y adaptar su modelo de trabajo hacia el trabajo en casa y aprender a compartir un espacio pequeño con sus familiares, las casas se convirtieron en lugares mixtos donde se realizan tanto tareas domésticas como actividades profesionales.

Esto derivo en un gran impacto en la vida de las personas, ya que es algo a lo que la mayoría no estaba acostumbrada.

Tobarra et ál. (2008) definen la usabilidad colaborativa, como un proceso eficaz, de satisfacción y eficiente para que un grupo en particular pueda lograr objetivos y metas en común en un contexto de uso específico de software en condiciones particulares de uso y coordinación.

Adicionalmente podemos encontrar soporte teórico y empírico que menciona que el éxito de las Mipymes está fuertemente relacionado con la habilidad que estas pequeñas y medianas empresas posean para la ejecución de actividades de colaboración internas y externas con otras empresas de manera eficiente y efectiva (Van de Vrande et ál., 2009).

Adicionalmente en su estudio relacionado con la colaboración Van de
Vrande et al. (2009) menciona que esta colaboración es fundamental para que las empresas adquieran nuevo conocimiento el cual es muy importante para que las Mipymes se mantengan en proceso de expansión y crecimiento constante, que esto será el detonante para una mejor gestión integral de las mismas y podrán incrementar sus ventajas competitivas y capacidades de innovación.

Bjorklund et ál. (2020) menciona la importancia de un ambiente colaborativo principalmente en tiempos de crisis, ya que los sistemas colaborativos en estos tiempos toman mayor relevancia, principalmente porque estos robustecen a los equipos de trabajo, favorece la creación de sinergias, se comparte conocimiento entre las partes y un punto importante para toda organización, es un ahorro en temas de costos.

Crick y Crick (2020) mencionan la importancia de la permanencia de los modelos de trabajo colaborativos una vez que la crisis sanitaria se encuentre controlada, esto es mantener la interacción colaborativa entre los miembros de los equipos de trabajo por medio de correos electrónicos, herramientas digitales y sistemas de gestión, ya que esto ayudara a mantener una comunicación eficiente dentro de la organización.

\subsubsection{Entornos colaborativos, estudios empíricos}

Honores et ál. (2020) realizaron una investigación de campo a través de la aplicación de encuestas, con el propósito de documentar información importante y necesaria para realizar un análisis relacionado con la capacitación del personal aplicada al trabajo colaborativo a través de los datos recolectados y desarrollados en el programa estadístico SPSS. Se consideró como universo de estudio la provincia de El Oro, con personal dedicado a la minería, los cuales de acuerdo con reporte elaborado por el Banco Central del Ecuador en el periodo 2017 existían 6,745 trabajadores en activo. Para el desarrollo de la investigación se consideró una muestra de 365 empleados, que fue el resultado de la aplicación de la fórmula de población finita. Los resultados obtenidos por Honores et ál. (2020) concluyen que el efecto de la 
capacitación del personal influye en el desempeño de las funciones relacionadas con el trabajo colaborativo de los trabajadores mientras efectúan sus funciones en la organización, ya que este tipo de programas de capacitación influye un $67 \%$ por lo tanto se podría establecer que mediante la capacitación se mejora el rendimiento de las unidades de trabajo de la organización.

Morillo y Rodríguez (2020) apoyándose en el método de estudio de caso con enfoque interpretativo como método empírico de investigación, aplicando encuesta y seleccionando a los encuestados con método no probabilístico intencional, teniendo como sujetos de estudio a directivos de mando medio concluyeron que el uso apropiado de tecnologías de información (TI) colaborativas están fuertemente asociadas al desempeño de los colaboradores de las empresa y adicionalmente estas TI colaborativas impactan de manera positiva en la comprensión de la información proporcionada por los empleados de la empresa.

Viveros y Camargo (2020) emplearon una metodología empleada para su investigación de tipo descriptivo - explicativo de índole documental, fundamentada en fuentes de carácter investigativo y desarrollos aplicativos para identificar los nuevos desafíos que tienen los administradores de empresas frente a la administración colaborativa. En lo referente a los materiales de recolección de datos se utilizó un compendio de información documental consultando fuentes de información secundaria. Entre los principales resultados se encontró que el desarrollo empresarial es más efectivo si los esfuerzos grupales enfocan su esfuerzo en integrar una administración colaborativa, ya que esta es una eficiente herramienta en la gestión organizacional.

Del Solar et ál. (2021) realizan un análisis cuantitativo basado en encuesta, la encuesta tiene diseño descriptivo, observacional, prospectivo y transversal. La búsqueda se realizó con información de tipo secundaria, documental y bibliográfica principalmente. Como principales resultados de la investigación se encontró que la aplicación de prácticas como informar a los trabajadores sobre los beneficios de utilizar el sistema Last Planner System (LPS) como herramienta de trabajo colaborativo deriva en importantes ahorros económicos para la empresa.

\subsection{Innovaciones, una visión teórica}

De acuerdo con Yoguel y Boscherini (1996), mencionan que la capacidad de innovación de las organizaciones es indispensable para mantener su ventaja competitiva vigente ya que esta es fundamental para la diferenciación de sus productos o servicios en un mundo cada vez más globalizado. La innovación es un proceso que deriva en la aplicación de una nueva idea (García et ál., 1999), es un término muy amplio que no se restringe al ámbito de las tecnologías o al uso e incorporación de las tecnologías de información y comunicación (TIC).

Alaya, Fernández y González (2004) mencionan que la innovación también incluye la mejora en productos y/o servicios, estas mejoras incluyen una mayor capacidad por parte del personal de la organización por lo que deben de contar con excelentes competencias ya que la innovación implica adquisición de nuevos conocimientos los cuales se adquieren por medio de capacitaciones en áreas de ventas, producción, administración, etc.

En las naciones en proceso de desarrollo y en las economías emergentes es muy importante identificar las principales variables que modifican el crecimiento de las economía local y nacional (Vrgovic et ál., 2012). De entre todas estas variables encontradas en las diferentes fuentes literarias podemos encontrar la innovación que es calificada por una gran cantidad de investigadores y académicos como la variable que mayormente influencia de manera positiva el crecimiento de las empresas (Jaffe y Lerner, 2006).

La innovación es un pilar para la competitividad de las Mipymes, su importancia radica en factores como el aumento de la competencia global, la baja duración de los ciclos de vida de productos y los cambiantes patrones de consumo de los clientes y comportamiento de los proveedores. 
Las grandes empresas definitivamente tienen un papel fundamental en la innovación, pero esto no quiere decir que no hay lugar para las Mipymes, ya que su esperanza de vida depende mucho de su adaptación al entorno y mercado actual (Markatou, 2012).

De acuerdo con la OCDE, en $1934 \mathrm{~J}$. Schumpeter menciono que la innovación es un proceso dinámico en el que las nuevas tecnologías sustituyen a las viejas tecnologías, a lo que él llamó destrucción creativa. Más adelante reconsidero su posición inicial y en el año de 1978 la rediseñó para referirse a la innovación como el ingreso de un nuevo producto, de un nuevo modelo de fabricación o la entrada a nuevos mercados, es decir, cualquier forma nueva y diferente de hacer las cosas (Escandón y Hurtado, 2014).

El tema de la innovación en las Mipymes no es tema nuevo, como lo mencionan Ospina y Puche (2014) quienes citan a Ruiz y Mandado (1989) "innovar en las Mipymes significa mejorar en la eficiencia operativa a través de inversión en tecnologías de producción, incrementando así los ingresos por productos nuevos o mejorados".

La inclusión de procesos de innovación en el modelo de negocio de las empresas origina la creación de una cultura hacia la creatividad e innovación que explora en las organizaciones no sólo el diseño y rediseño de nuevos procesos, sino también la mejora continua y adaptación a los cambios que los nuevos ambientes requieren (García, 2019).

Ibarra et ál. (2020) menciona la importancia de las innovaciones en las Mipymes ya que estas innovaciones aseguran que los modelos de negocio tengan éxito desde su implementación.

Putra et ál. (2020) menciona la importancia de la innovación y su relación con el éxito de las organizaciones durante la pandemia, principalmente por la importancia que tiene el que la empresa pueda adaptarse en tiempo y forma para enfrentar estas crisis, en la mayoría de los casos se tiene que evolucionar e implantar productos y servicios nuevos que den un valor agregado a los clientes en estos tiempos de contingencia sanitaria.
Reeves y Fuller (2020) mencionan que ante la actual pandemia por Covid-19 las organizaciones deben de tener la capacidad de reaccionar rápidamente ante los cambios, esto incluye el desarrollar nuevas estrategias de adaptación e innovación de procesos. La innovación debe ser la punta de lanza de los emprendedores ya que tiempos como los que se viven actualmente generan cambios en la forma que se consumen los productos y servicios, siempre es importante la generación de valor más aun en estos momentos.

\subsubsection{Innovaciones, estudios empíricos.} Cando et ál. (2020) metodológicamente fundamentaron su investigación siendo esta de tipo descriptiva con diseño de campo no experimental transversal, desarrollada en una población de 56 emprendedores en la categoría de restaurantes de la ciudad de Macas, provincia de Morona Santiago, Ecuador, aplicando encuestas en línea de tipo cuestionario de 12 preguntas validadas por juicio de expertos y cálculo de alfa de Cronbach de 0.89. En la investigación evidenciaron que la innovación es fundamental en las Mipymes durante la actual crisis sanitaria y las que pudieran presentarse en un futuro, con ello es esencial desarrollar una nueva experiencia administrativa para ser aplicada. El desarrollo de un procedimiento estratégico permitirá a los administradores de las empresas gestionar las actividades con mayor eficiencia.

Montalvo y Orozco (2020) desarrollando una metodología de tipo cualitativa, con un enfoque interpretativo, de carácter documental bibliográfica, utilizando como técnica principal la recopilación de la información y recurriendo a búsquedas avanzadas de descriptores clave con una población total de 44 documentos que le consintieron desarrollar la investigación con un muestreo intencional un total de 09 documentos encontraron que el concepto disrupción ha sido últimamente asociado con las innovaciones y que este concepto se ha mantenido vigente principalmente a raíz de que entraron los modelos innovadores de empresas denominadas start-ups tecnológicas, entendida como una gran organización en su etapa inicial cuyo negocio, a diferencia de las 
Mipymes tradicionales, utiliza las tecnologías digitales como principal fuente de innovación para encumbrarse de forma más rápida y eficiente en el mercado.

Rodríguez y Tagle (2020) identifican metodológicamente tres momentos en su investigación los cuales son el encuadre teórico y la metodología de su análisis, el segundo fue la búsqueda de la información para conocer el impacto económico de la pandemia y el tercero se relacionó con la obtención de información primaria directamente de los sujetos de estudio. El instrumento utilizado fue la aplicación de encuestas en donde los resultados arrojaron conclusiones como que los empresarios deben de replantearse las estrategias para ser más competitivos y que la innovación como una de sus variables investigadas otorga nuevas oportunidades para mitigar riesgos ocasionados por la pandemia actual.

Se incluye el trabajo realizado por García, Grilló y Morte (2021) en donde seleccionaron 28 artículos de las siguientes bases de datos científicas, Scopus y Web of Science, utilizando el diagrama de flujo de decisiones de inclusión propuesto por PRISMA (Preferred Reporting Items for Systematic Reviews and Meta-Analysis), como principal descubrimiento relacionado con la innovación obtenemos indicar que la adopción de innovaciones conexas con la propuesta de valor, en la gran mayoría de las ocasiones se manifiesta forma de nuevos productos y servicios, como forma más común de experimentación de cara al consumidor y la generalización de iniciativas de colaboración entre todos los participantes del ecosistema empresarial.

Por último, Pérez, García y García (2021) incluyen en su estudio la variable innovación, donde su indicador formativo son las actividades en $\mathrm{I}+\mathrm{D}+\mathrm{i}$ ya que estas son consideradas de manera integral como promotoras de la innovación (López-Mielgo et al., 2012). La cuantificación de los análisis elaborados a través de la encuesta tipo Likert de 1 a 3 niveles siguió el siguiente patrón: realización de actividades de manera normal, sin realizar esfuerzo o actividades adicionales y $\sin$ presupuestos $\mathrm{o}$ proyectos comprometidos de por medio. La hipótesis planteada fue $\mathrm{H} 2$ : Existe una relación positiva y significativa entre el esfuerzo en innovación de una empresa y por último su capacidad de adaptación ante el shock externo producido por la COVID-19. Las conclusiones de esta investigación sugieren que el rol mediador de la innovación en el ambiente actual es significativamente un factor determinante para el éxito empresarial en tiempos de pandemia.

\subsection{Nuevos modelos de negocio, una visión teórica}

La flexibilidad organizativa incorpora la habilidad de las empresas para modificar de manera eficiente, rápida y efectiva la adaptación a nuevos ambientes en entornos inciertos, todo esto aplicando al máximo los recursos y capacidades tanto de infraestructura como de capital humano. (Volberda, 1996). Para Osterwalder y Pigneur (2010) un modelo de negocio representa el fundamento natural de cómo una empresa crea, entrega y captura valor en sus procesos. Su enfoque ha difundido una representación de los modelos de negocio a través del modelo de negocio de Canvas, que incluye las áreas relacionadas con clientes, viabilidad económica, infraestructura y oferta.

Eisenmann (2011) menciona que un modelo de negocio es un conjunto compuesto de características que detallan la propuesta única de valor para el cliente de una empresa y cómo alinea sus acciones y las de sus colaboradores para entregar ese valor y obtener beneficios sostenibles.

La flexibilidad de la organización y su adaptación hacia nuevos modelos de negocio puede ser fragmentada en tres dimensiones (Sánchez et al., 2011), la primera seria la flexibilidad productiva que es la habilidad de las organizaciones para diversificar los productos o servicios que ofrecen. Por otro lado, encontramos la flexibilidad de la estructura organizativa que es la capacidad que tiene la estructura organizacional en adaptarse a nuevos modelos de negocio como la internacionalización, tamaño del negocio o el descentralizar actividades tanto administrativas como operativas. Por último, tenemos la flexibilidad laboral que es la habilidad de las áreas de capital humano para 
adaptarse a nuevas condiciones tanto externas como propias de la organización.

Es importante considerar lo comentado por Morejón, Hernández y Jaramillo (2014) donde aseguran que los modelos de negocio estáticos pueden ser peligrosos, ya que los modelos de negocio de las Mipymes bajo un contexto estratégico deben ser dinámicos y continuos.

Es importante la adaptación en los nuevos modelos de negocio la implementación del trabajo a distancia, aunque esta es una opción que no todas las organizaciones pueden implementar, para esto es importante que se realice un diagnostico e identificar los procesos que son viables realizarse desde casa (Giles, 2020).

Entender el entorno económico actual para descubrir los nuevos desafíos y oportunidades es el inicio para elaborar un plan estratégico que responda a las necesidades de permanencia de las organizaciones. Este plan debe incluir temas como la modificación en los patrones de consumo de los clientes, una nueva propuesta de valor, nuevos modelos financieros, canales de comercialización que se adapten al entorno actual y la apertura hacia nuevos canales online (KPMG, 2020).

La reinvención de los modelos de negocio es apremiante en estos tiempos de cambio, ya que facilita las relaciones interpersonales en beneficio de las personas, las organizaciones deben ver en esta reinvención una oportunidad para optimizar, aprender de otros, solucionar necesidades e innovar.

Seetharaman (2020) menciona la importancia que tiene el rediseñar los modelos de negocio para el éxito de la empresa en este nuevo ecosistema, principalmente adaptarse al tema de las nuevas tecnologías en donde el trabajo remoto juega un papel importante, adicional a estar presente en medios digitales con clientes y proveedores.

\subsubsection{Nuevos modelos de negocio, estudios empíricos}

Barrientos et ál. (2020) en su investigación de tipo descriptiva y de investigación aplicada ya que se fundamenta principalmente en evidencia tecnológica de investigación básica, realizando un proceso de enlace entre la teoría y el producto. La población objeto de estudio se conformó de 4 líderes en procesos de la panificadora, con una muestra reducida de 4 personas, todos empleados de la empresa. Por último, se utilizó una técnica de recolección de información mediante dos métodos, la entrevista (aplicada a la muestra seleccionada) y el otro método es la observación, donde se obtuvieron datos que proporcionaron elementos de apoyo para la investigación. Como principales hallazgos en la investigación se encontró que el cambio del modelo de negocio tradicional (mecánico) a la conversión de planta inteligente dejo en claro que al automatizar procesos se genera un mayor volumen de datos lo que da como resultado un mejor manejo integral de recursos lo que da como consecuencia mayor eficiencia y mejora en resultados para la panificadora, el involucramiento de la tecnología hace más productiva y competitiva a la empresa para hacer frente a los desafíos que trajo consigo la pandemia del SARS-CoV-2.

Hoyos y Sastoque (2020) presentaron en su artículo realizado a partir de fuentes bibliográficas con referentes nacionales e internacionales, un enfoque de revisión documental, evaluado a partir de su contribución para dar respuesta a su pregunta ¿Es el Marketing Digital la oportunidad de digitalización de las PYMES en Colombia en tiempo del Covid-19? Se encontró que el marketing digital como innovación en los modelos de negocio puede llegar a ser un aliado y una oportunidad de desarrollo para las Mipymes durante los tiempos de contingencia sanitaria y se quedaran una vez que esta sea controlada ya que este proceso incluirá la utilización de plataformas tecnológicas y se aprovecharan herramientas y elementos del marketing digital en la cadena de valor, como también otros procesos industriales, se podrá incluir la inteligencia artificial, tecnología Blockchain, internet de las cosas, etc.

Ordoñez, Narváez y Erazo (2020) en su investigación desarrollaron una representación descriptiva, investigando y profundizando sobre la problemática 
relacionada con los sistemas financieros, emplearon un diseño transaccional no experimental, empleando para la recolección de datos técnicas de investigación como la entrevista y la encuesta, aplicando cuestionarios para recabar información relevante. El universo de estudio lo conformaron 6 instituciones financieras localizados en la ciudad de Cuenca. Como principal conclusión se determinó que la inserción de modelos de negocios no sólo explora las demandas actuales del mercado financiero, sino que se ajusta a las necesidades económicas de las empresas para dinamizar la económica que atiende su mercado para de esta forma enfrentar cambios, crisis emergentes como la pandemia por coronavirus (COVID-19).

Palomino, Mendoza y Oblitas (2020) decidieron utilizar la técnica del cuestionario en línea estructurado y auto - administrado, utilizaron un método no probabilístico y de accesibilidad, con los datos obtenidos a través de un cuestionario realizado y puesto a disposición en una plataforma en línea (SurveyMonkey), el instrumento para recabar la información lo validaron a través de un CFA (Confirmatory Factor Analysis), logrando una confiabilidad de 0.856 y una alfa de Cronbach de 0.913. El cuestionario que utilizaron incluía 9 preguntas sociodemográficas, 8 relacionadas con la compra en línea, 9 sobre las compras antes y después del Covid-19 y finalmente 2 sobre la intención de compra antes y después del Covid-19. Como principales conclusiones se evidencia que los nuevos modelos de negocio orientados a las compras en línea habrán de poseer la capacidad de proveer a sus consumidores y crear en ellos buenas y nuevas experiencias. El e-commerce se ha convertido en una necesidad actual en los clientes, los nuevos modelos de negocio de las empresas en temas de comercio en línea deberán de representar experiencias para que los consumidores mantengan la tendencia del ecommerce. El éxito en los modelos de negocio electrónico dependerá de las estrategias de marketing que cada empresa logre diseñar e implementar de manera integral incluyéndola en la cadena de valor siendo un diferenciador contra su competencia.

En la figura 1 encontramos los diferentes factores que influyen en las Mipymes en tiempos de pandemia, se observan algunos de los autores que han investigado y definido cada una de estas variables comentadas anteriromente.

Es importante mencionar que se realizo una revision exhaustiva de literatura e investigaciones empiricas, derivado de eso se incluyeron a los autores que se considero realizaron mayor aporte al tema de estudio.

\section{MÉTODO}

El presente estudio es de tipo documental ya que se realizaron consultas en diferentes instrumentos como revistas, investigaciones empíricos, registros y diferentes fuentes existentes y bibliográficas ya que se revisó material bibliográfico existente respecto a nuestro tema de investigación.

Una vez conseguida la información, se procedió a elaborar un análisis de datos con la intención de estudiar el comportamiento de las diferentes variables en cuestión y obtener las principales aportaciones de cada una de las investigaciones empíricas analizadas para entender los beneficios que estas variables tienen en las Mipymes.

Figura 1. Modelo Teórico - Factores que Influyen en las en las Mipymes en Tiempos de Pandemia.

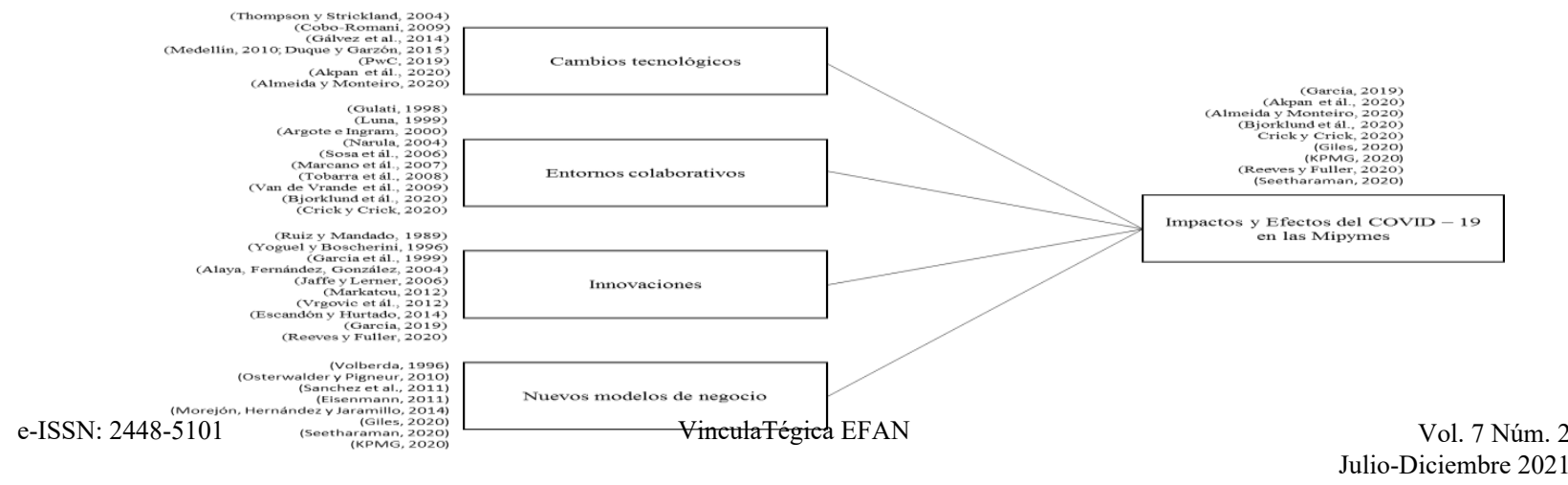


Fuente: Autores citados, elaboracion propia.

\section{RESULTADOS}

Es importante analizar el comportamiento que tienen las Mipymes en tiempos de contingencia sanitaria y encontrar los factores que influyen en estas en tiempos de pandemia. A continuación se presentaran los principales resultados obtenidos que cada una de nuestras variables objeto de análisis.

En el caso de nuestra variable cambios tecnológicos enfocado en el uso de las TIC los principales resultados encontrados fueron que al hacer uso de las TIC para temas de promoción y publicidad de productos y servicios estas influyen de manera positiva en los resultados de las Mipymes (Bermeo, Montoya y Mejía, 2020). Por otro lado se confirma la generación de nuevas oportunidades de mercado al implementar nuevas tecnologías (Heredia, Aguilar y Sainz, 2020). Otro hallazgo realizado fue la importancia del e-commerce en tiempos de pandemia en donde Rodríguez et ál., (2020) confirman que el e-commerce funciona como catalizador para la comercialización de productos y servicios para las Mipymes. Por último Pérez, García y García, 2021 mencionan que las TIC son un factor determinante para la administración de información y coordinar actividades profesionales de manera eficiente e tiempos de contingencia sanitaria.

En el caso de la variable entornos colaborativos se encontró que la capacitación es un factor determinante para mejorar el desempeño laboral del personal al realizar actividades colaborativas dentro de la organización (Honores et ál., 2020). Adicionalmente se encuentra como principal resultado que el uso apropiado de las TIC colaborativas para cubrir las necesidades de la organización y como herramienta para el desarrollo de nuevos modelos colaborativos organizacionales incrementan la eficiencia, mejoran tiempos de respuesta y mejoran las relaciones con clientes para incrementar sus ventas (Morillo y Rodríguez, 2020).

Nuestra siguiente variable nos muestra resultados y nos menciona según Tagle, Granados y Rodríguez (2020) que la innovación utilizada por las Mipymes en tiempos del Covid-19 es una estrategia para incrementar la competitividad de las organizaciones y para mitigar riesgos derivados de contingencias sanitarias.

En el caso de Cando, et ál., (2021) su principal hallazgo fue el que la innovación es un factor fundamental para las Mipymes en tiempos de crisis sanitaria y que el desarrollo de procedimientos estratégicos les permitirán a los administradores gestionar actividades con mayor eficiencia. Por otro lado Pérez, García y García (2021) mencionan que la innovación es un mediador determinante para el éxito de las empresas en tiempos de pandemia.

Por último los resultados encontrados en tema de modelos de negocio fueron que al modificar modelos de negocio mecánicos (tradicionales) a modelos de negocio inteligentes (industria 4.0) las empresas mejoran considerablemente resultaos en temas de fabricación en sus plantas productivas (Barrientos et ál., 2020). Otro resultado importante fue el determinado por Hoyos y Sastoque (2020) donde determinan que al migrar su modelo de negocio hacia la implementación de marketing digital y digitalización esto influyo de manera positiva en mejorar tiempos de respuesta con clientes y proveedores, adicional a que lograron tener una mayor penetración en el mercado nacional.

Ordoñez, Narvaez y Erazo (2020) concluyeron en su estudio que la utilización del sistema financiero como nuevo modelo de negocio ayuda positivamente para la modernización y realización de inversiones en las empresas ya que estos recursos adelantarían inversiones que no se podrían lograr con los propios flujos de la empresa, 
adicionalmente los flujos se verían favorecidos al poner en marcha los equipos adquiridos producto de estas nuevas inversiones.

Finalmente se determina que la inclusión del e-commerce en los modelos de negocio actuales genera valor en canales de venta, tecnología y publicidad de acuerdo, lo que da como resultado una mejoría en márgenes de venta, esto de acuerdo a investigación realizada por Palomino, Mendoza y Oblitas (2020).

\section{DISCUSION Y CONCLUSIONES}

En la presente investigación se han explorado algunos de los principales factores que las Mipymes han tenido que modificar, replantear o adaptar a raíz de la pandemia por Covid-19, se ha encontrado que dentro de estos factores de adaptación algunos son los cambios tecnológicos, los entornos colaborativos, las innovaciones y la aplicación de nuevos modelos de negocio. Lo antes mencionado se relaciona directamente con muchas de las Mipymes ya que estas fungen como proveedores de todos estos sectores económicos, de ahí deriva la importancia de conocer todos los sectores económicos afectados por la pandemia actual originada por el Covid-19.

Los efectos que ha tenido la pandemia generada por el coronavirus en las empresas son muy importantes, actualmente se vive una de las peores crisis en los últimos años, hemos visto una disminución en las actividades consideradas como no esenciales por los gobiernos, miles de empresas han cerrado, altos porcentajes de desempleo a nivel mundial, baja en las relaciones comerciales entre países, por estas razones las Mipymes no pueden dejar de lado temas como la los cambios tecnológicos, los entornos colaborativos, innovación y la constante actualización de sus modelos de negocio, todo esto en beneficio y apoyo a su supervivencia principalmente en tiempos de pandemia.

Como conclusión relacionada con los cambios tecnológicos en tiempos de pandemia podemos mencionar que estos llegaron para quedarse, temas como el de mejorar la infraestructura tecnológica de las Mipymes nunca fueron tan relevantes e indispensable como en esta nueva normalidad, adicionalmente el automatizar procesos ha ayudado a que temas como el contagio en los empleados se reduzca para un eventual regreso a las labores en las empresas. El uso de dispositivos digitales, conexiones de calidad y herramientas para el trabajo remoto serán parte de nuestro nuevo día a día. Como principal conclusión relacionada con los entornos colaborativos podemos mencionar que estos apoyan fomentando el intercambio de ideas, promueven la innovación, optimizan los recursos de las organizaciones y reducen los errores, todo esto en beneficio de las empresas.

Es importante mencionar que las micro, pequeñas y medianas empresas de diferentes países alrededor del mundo, especialmente en países en vías de desarrollo, muestran que las actividades de colaboración e interacción que tienen las empresas con otras organizaciones son cada vez más importantes, no solamente para que las Mipymes puedan aumentar sus actividades de innovación, sino también para mejorar la promoción e incremento de las habilidades de innovación (Biggs y Shah, 2006; Liefner, Hennenmann y Xin, 2006).

La innovación en tiempos de pandemia ha representado todo un reto para las Mipymes, aunque estas se caracterizan por ser innovadoras por naturaleza, es importante que mantengan esta innovación en constante desarrollo aplicándola en la mejora de procesos, desarrollo y aplicación de nuevas tecnologías así como incorporando programas incentivando procesos innovadores, recordar a la innovación constante como ventaja competitiva. En relación a los modelos de negocio se concluye que las Mipymes se han visto forzadas a modificar y replantear sus modelos de negocio ante la contingencia sanitaria, es importante mencionar que estas modificaciones a las que se vieron obligadas a realizar las empresas tarde o temprano se tendrían que realizar como por ejemplo el trabajo a distancia y la flexibilidad laboral, este se ha incrementado ya que ha sido una solución para evitar contagios masivos del virus COVID-19 en las empresas, la incursión en la era de la industria 4.0, el marketing 
digital, la utilización del sistema financiero digital y la incursión de modelos de negocio dirigidos hacia el e-commerce.

La presente investigación nos deja mucho campo de estudio para realizar futuras investigaciones relacionadas con los elementos que influyen de manera positiva en las Mipymes a raíz de crisis y contingencias sanitarias por lo que más adelante se continuara bajo la línea de investigación relacionada a las Mipymes y los efectos que los diferentes entornos las afectan manera positiva como negativa.

En la figura 2 podemos visualizar los efectos económicos derivados del Covid-19, en donde encontramos efectos directos en otros sistemas como lo son los de salud, distanciamiento social, efectos en la oferta y demanda de productos y servicios, afectaciones directas en temas de educación, turismo, manufactura, etc., adicional se ven los efectos de mediano y largo plazo entre los que se encuentran quiebras de empresas, menos inversión privada, aumento de desempleo, menores salarios, etc.

Lo antes mencionado se relaciona directamente con investigaciones futuras que se pueden desarrollar relacionadas con las Mipymes y su entorno ya que estas fungen como clientes y proveedores de todos estos sectores económicos, de ahí deriva la importancia de mantener esta línea de investigación vigente.

Figura 2. Efectos económicos derivados del Covid-19.

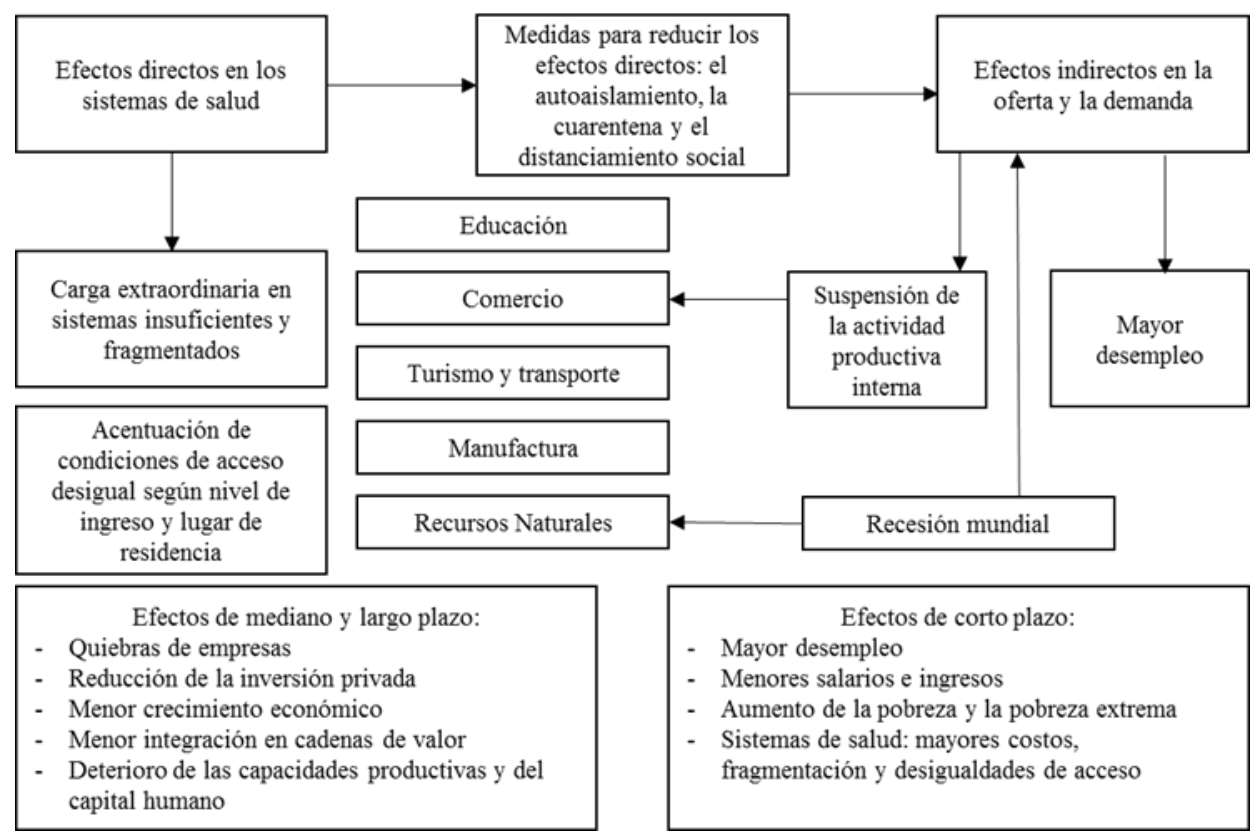

Fuente: Comisión Económica para América Latina y el Caribe (CEPAL, 2020a). 


\section{REFERENCIAS}

Akpan, I.J., Soopramanien, D., \& Kwak, D.H. (2020). Cutting-edge technologies for small business and innovation in the era of COVID-19 global health pandemic. Journal of Small Business \& Entrepreneurship, 1-11. https://doi.org/10.1080/08276331.2020.1799294

Alaya, J., Fernández, R., \& González, M. (2004). Capacidades tecnológicas y certificaciones de calidad: Aplicación empírica a las pymes de Rioja. Cuadernos de Gestión, 69-82.

Almeida, F., Santos, J.D., \& Monteiro, J. A. (2020). The challenges and opportunities in the digitalization of companies in a post-COVID-19 World. IEEE Engineering Management Review, 48(3), 97-103. https://doi.org/10.1109/EMR.2020.3013206

Argote, L. y Ingram, P. (2000). Knowledge transfer: A basis for competitive advantage in firms. Organizational Behaviourand Human Decision Processes, 82, 150-169.

Barrientos, E., Areniz, Y., Coronel, L., Cuesta, F. y Rico, D. (2020). Modelo de incursión en la industria 4.0 aplicado a la compañía alimenticia tu pan gourmet SAS: Estrategia para el renacer en la pandemia ocasionada por COVID-19 (SARS-CoV-2). Revista Ibérica de Sistemas e Tecnologías de Informacao.

Bermeo-Giraldo, M.C., Montoya-Restrepo, L.A., Valencia-Arias, A., y Mejía Cardona, M.A. (2020). Incursión de las TIC en la gestión de la información financiera en las empresas pyme comerciales: estudio de caso. NOVUM, 1(10), $25-41$.

BID. (2020). Respuestas al COVID-19 desde la ciencia, la innovación y el desarrollo productivo, Washington DC, Banco Interamericano de Desarrollo. https://publications.iadb.org/publications/spanish/document/Respuestas-al-COVID-19desde-laciencia-la-innovacion-y-el-desarrollo-productivo.pdf.

Biggs, T. y Shah, M. K. (2006). African SMEs, networks, and manufacturing performance. Journal of Banking and Finance, 30(11), 3043-3066.

Bjorklund, T.A., Mikkonen, M., Mattila, P., \& van der Marel, F. (2020). Expanding entrepreneurial solution spaces in times of crisis: Business model experimentation amongst packaged food and beverage ventures. Journal of Business Venturing Insights, 14, e00197.https://doi.org/10.1016/j.jbvi.2020.e00197

Campbell, M.C., Inman, J.J., Kirmani, A., \& Price, L.L. (2020). In times of trouble: A framework for understanding consumers' responses to threats. Journal of consumer research, 47(3), 311326.https://doi.org/10.1093/jcr/ucaa036

Cando, A., Erazo, J., Álvarez, Juan. y Rivera, J. (2021). La pandemia COVID- 19 en la microempresa: Emprendimiento e Innovación. Revista Interdisciplinaria de Humanidades, Educación, Ciencia y Tecnología. DOI 10.35381/cm. v7i12.445

CEPAL. (2020). Euro Mi Pyme - COVID-19. https://www.cepal.org/es/euromipyme/mipymescovid-19

CEPAL. (2020a). América Latina y el Caribe ante la pandemia del COVID-19: efectos económicos y sociales. Editorial CEPAL. Disponible en https://www.cepal.org/es/publicaciones/45337america-latina-caribe-la-pandemia-covid-19-efectos-economicos-sociales

Chiao, Y.-C., Yang, K.-P., \& Yu, C.-M. J. (2006). Performance, Intenatinalization, and Firm-specific Advantages of SMEs in a Newly Industrialized Economy. Small Business Economics, 26(5), 475-492.

Cobo-Romani, J. C. (2009). El concepto de tecnologías de la información. Benchmarking sobre las definiciones de las TIC en la sociedad del conocimiento. ZER-Revista de Estudios de Comunicación, 14(27), 295-318. https://bit.ly/2NoSUrL

Crick, J.M., \& Crick, D. (2020). Coopetition and COVID-19: Collaborative business-to-business marketing strategies in a pandemic crisis. Industrial Marketing Management, 88, 206-213. https://doi.org/10.1016/j.indmarman.2020.05.016

Dannenberg, P., Fuchs, M., Riedler, T., \& Wiedemann, C. (2020). Digital transition by COVID-19 pandemic? The German food online retail. Tijdschrift voor economische en sociale geografie, 
111(3), 543-560. https://doi.org/10.1111/tesg.12453

Del Solar, P., Del Rio, M., Fuente, R., y Esteban, C. (2021). Herramientas de trabajo colaborativo en el sector de la construcción español. Buenas prácticas para la implementación de la metodología "Último Planificador (LPS)". Informes de la Construcción, 73(561), e383-e383.

Duque Oliva, E. J. y Garzón, A. G. (2015). Adaptación de las organizaciones a los cambios del entorno, SUMA NEG.; 6 (13): 1

Eisenmann, T. (2011). Business Model Analysis, Part 1: Key Questions. Recuperado en http://platformsandnetworks.blogspot.mx/2011/07/businessmodel-analysis-part-1-key.html

El Heraldo de México. (2020). Impacto del COVID-19 en las PYMES. El Heraldo de México. https://heraldodemexico.com.mx/opinion/2020/4/9/impacto-del-covid-19-en-las-pymes166447.html

Escandón, M., y Hurtado, A. (2014). Los Determinantes de la Orientación Exportadora y los Resultados en las Pymes Exportadoras en Colombia. Estudios Gerenciales, 430-440.

Gálvez, E., Riascos, S. y Contreras, F. (2014). Influencia de las tecnologías de la información y comunicación en el rendimiento de las micro, pequeñas y medianas empresas colombianas. Estudios Gerenciales, (133), 355-364. doi: 10.1016/j.estger.2014.06.006

Gamero, H., \& Ostos, J. (2020). Revisión sistemática de literatura sobre factores clave en la identificación de oportunidades de negocio. Retos Revista de Ciencias de la Administración y Economía, 10(20), 307-327. https://doi.org/10.17163/ret.n20.2020.07.

García, K. (2019). Gestión de la innovación. Una visión de modelo de negocio. K-Consensus. Revista de publicaciones Científicas y Académicas, 3(3), 3-16.

García, L.F., Mareo, L.B., Molina A., J.F., y Quer, R.D. (1999). La capacidad innovadora como intangible empresarial: una aproximación a través de la gestión del conocimiento. Revista Espacios, Vol. 20(3), 1-4.

García-Madurga, M.A., Grillo-Méndez, A.J., y Morte-Nadal, T. (2021). La adaptación de las empresas a la realidad COVID: una revisión sistemática. Retos Revista de Ciencias de la Administración y Economía, 11(21), pp. 55-70. https://doi.org/10.17163/ret.n21.2021.04

Giles-Navarro. C. (2020). Recomendaciones para las MIPyME ¿Qué hacer para sobrevivir a la pandemia del Covid-19? Recommendations for MSMEs What to do to survive the Covid-19 pandemic? Instituto Belisario Domínguez Senado de la República. Recuperado de https://n9.cl/gi4h

González, F. (2020). Las tendencias del consumidor que definirán la nueva normalidad. Merca2.0. https://www.merca20.com/tres-tendencias-del-consumidor-que-definiran-lanuevanormalidad/

Gruszczynski, L. (2020). The COVID-19 pandemic and international trade: Temporary turbulence or paradigm shift? European Journal of Risk Regulation, 11(2), 337-342. https://doi.org/10.1017/err.2020.29

Gulati, R. (1998). Alliances and networks. Strategic Management Journal, 19(4), 293-317.

Heredia, J., Aguilar, P. y Sainz, N. (2020). Situación de las Pymes de Hermosillo, Sonora ante la crisis presentada por COVID-19. Revista de Investigación Académica Sin Frontera. https://revistainvestigacionacademicasinfrontera.unison.mx/index.php/RDIASF/

Honores, N., Vargas, C., Espinoza, C., \& Tapia, N. (2020). Importancia y capacitación personal: aprendizaje colaborativo y desempeño laboral en las empresas mineras. 593 Digital Publisher CEIT, 5(6), 398-409.

Hoyos-Estrada, S., y Sastoque-Gómez, J. (2020). Marketing Digital como oportunidad de digitalización de las PYMES en Colombia en tiempo del Covid - 19. Revista Científica Anfibios, 3(1), 39-46. https://doi.org/10.37979/afb.2020v3n1.60

Ibarra, D., Bigdeli, A.Z., Igartua, J.I., \& Ganzarain, J. (2020). Business model innovation in established SMEs: A configurational approach. Journal of Open Innovation: Technology, Market, and Complexity, 6(3), 76. https://doi.org/10.3390/joitmc6030076

INEGI. (2020). Censos Económicos 2019. Resultados oportunos. Disponible en: 
https://www.inegi.org.mx/contenidos/programas/ce/2019/doc/pro_ce2019.pdf

Jaffe, A. y Lerner, J. (2006). Innovation and its discontents. En A. Jaffe, J. Lerner, y S. Stern (Eds.), Innovation policy \& the economy. Cambridge, MA: MIT Press.

Katua, N. T. (2014). The Role of SMEs in Employment Creation and Economic Growth in Selected Countries. International Journal of Eduacation and Research., 12(2), 461-472.

KPMG. (2020). Los cambios en los modelos de negocio muestran nuevas perspectivas para el sector minorista. Consultado el 21 de abril del 2021. Disponible en https://home.kpmg/ar/es/home/media/press-releases/2020/07/los-cambios-en-los-modelosde-negocio-muestran-nuevas-perspectiv.html

Liefner, I., Hennenmann, S. y Xin, L. (2006). Cooperation in the innovation process in developing countries: Empiricalevidence from Zhongguancun, Beijing. Environment and Planning A., 38(1), 111-130.

López-Mielgo, N., Montes-Peón, J.M., y Vázquez-Ordás, C. (2012). ¿Qué necesita una empresa para innovar? Investigación, experiencia y persistencia. Revista Europea de Dirección y Economía de la Empresa, 21(3), 266-281. https://doi.org/10.1016/j.redee.2012.05.005

Luna, C. (1999). Ingeniería Simultánea - Un Enfoque Para Reducir Los Tiempos De Entrega, La Mejora De La Calidad Y Disminuir Los Costos. Ingeniería \& Desarrollo, 5, 80-91.

Marcano A., Yelitza J. y Talavera P. (2007). Metodologías para el desarrollo de ambientes de aprendizaje en entornos colaborativos: Una reflexión teórica. Multiciencias, 7(1) ,63-71. Fecha de Consulta 6 de mayo de 2021. ISSN: 1317-2255. Disponible en: https://www.redalyc.org/articulo.oa?id=90470108

Markatou, M. (2012). The Role and the Importance of the Greek SMEs in the Production of Innovation. National and Kapodistrian University of Athens. Technological Education Institute of Larissa, Larissa, Greece.

Medellín Cabrera, E. A. (2010). Gestión tecnológica en empresas innovadoras mexicanas. RAI Revista de Administración e Innovación, 7(3), 58-78

Montalvo, L. y Orozco, C. (2020). Disrupción digital en tiempos de pandemia efectos en el mercado tecnológico en la provincia de Manabí - Ecuador. Polo del Conocimiento. DOI: 10.23857/pc. v5i8.1592

Morejón, V. M. M., Hernández, L. J. G., \& Jaramillo, V. V. S. (2014). Modelo de negocios de las Pyme: Un análisis de sus manejos financieros. Revista Raites, 7(13), 69-82.

Morillo, C. R., y Rodríguez, V. R. M. (2020). Desempeño empresarial con el uso de tecnologías de la información colaborativas: un estudio de caso en el sector automotriz. REVISTA CIENTÍFICA ECOCIENCIA, 7(4), 50-72.

Narula, R. (2004). R\&D collaboration by SMEs: New opportunities and limitations in the face of globalization. Technovation, 25, 153-161.

Ordoñez, E., Narvaez, C. y Erazo, J. (2020). El sistema financiero en Ecuador. Herramientas innovadoras y nuevos modelos de negocio. Revista Arbitrada Interdisciplinaria KOINONIA. http://dx.doi.org/10.35381/r.k.v5i10.693

Ospina, M. y Puche, M. (2014). Gestión de la Innovación en Pequeñas y Medianas Empresas. Generando ventajas competitivas y posicionamiento en el Mercado. Revista GPT Gestión de las Personas y Tecnología. Consultado el 21 de abril del 2021. Disponible en https://dialnet.unirioja.es/servlet/articulo?codigo=4760745

Osterwalder A. y Pigneur, Y. (2010). Business Model Generation: A Handbookfor Visionaries, Game Changers, and Challengers

Palomino, A., Mendoza, C. y Oblitas, J. (2020). E-commerce y su importancia en épocas de COVID19 en la zona norte del Perú. Revista Venezolana de Gerencia. DOI:10.37960/rvg. v25i3.33367

Perales, M. (2020). Experto Tec comparte las 10 tendencias de consumo que les permitan a pequeñas y medianas empresas conectar con su mercado meta en la nueva normalidad de consumo. 
https://tec.mx/es/noticias/estado-de-mexico/emprendedores/las-10-tendencias-deconsumopara-el-resurgimiento-de-las

Pérez-Calle, R., García-Casarejos, N. y García-Bernal, J. (2021). La empresa española ante la COVID-19: factores de adaptación al nuevo escenario. Revista de Ciencias de la Administración y Economía, 11 (21), pp. 5 - 24. https://doi.org/10.17163/ret.n21.2021.01

Putra, I., Sunarsih, N., Novitasari, L., y Setini, M. (2020). Exploring the relationship between social capital, innovation capability and innovation during the coronavirus pandemic. Uncertain Supply Chain Management, 8(4), 857-864. http://dx.doi.org/10.5267/j.uscm.2020.5.007

PwC, España. (2019). European Private Business Survey. Disponible en https://www.pwc.es/es/publicaciones/economia/european-private-businesssurvey-2019.html

Reeves, M. y Fuller, J. (2020). We Need Imagination Now More Than Ever. Harvard Business Review Home, 10 de abril de 2020. Disponible en: https://hbr.org/2020/04/weneedimagination-now-more-than-ever

Rodríguez, K., Ortiz, O., Quiroz, A. y Parrales, M. (2020). El e-commerce y las Mipymes en tiempos de COVID-19. Revista Espacios, Especial COVID-19, Vol. 41 (42) 2020. Art.9.

Rosales, R. (2020). La Pyme Post Covid 19: ¿Seguirá Siendo un Comodín entre el Estado y el Mercado? Catalejo. Un espacio para los apasionados por el conocimiento. www.catalejovirtual.com

Ruiz, M. y Mandado, E. (1989). La innovación tecnológica y su gestión. Productica, Marcombo S.A 1989, España.

Sánchez, A.M., Jiménez, M.J.V., y Pérez, M.P. (2011). Innovación y flexibilidad de recursos humanos: el efecto moderador del dinamismo del entorno. Revista Europea de Dirección y Economía de la Empresa, 20(1), 41-68.

Seetharaman, P. (2020). Business models shifts: Impact of Covid-19. International Journal of Information Management. 54, 102173. https://doi.org/10.1016/j.ijinfomgt.2020.102173

Segura, O. (2020). El Coronavirus y la Sociedad Global del Riesgo. Economía y COVID - 19. Campus. Oficina de Comunicación. Universidad Nacional. Obtenido de http://publica2.una.ac.cr/periodicoCampus/abril-2020/pdf/campuscriterios.pdf

Sharma, P., Leung, T. Y., Kingshott, R. P. J., Davcik, N. S. y Cardinali, S. (2020). Managing uncertainty during a global pandemic: An international business perspective. Journal of Business Research, 116, 188-192. https://doi.org/10.1016/j.jbusres.2020.05.026

Sheth, J. (2020). Impact of Covid-19 on Consumer Behavior: Will the Old Habits Return or Die? Journal of Business Research, 117, 280-283. https://doi.org/10.1016/j.jbusres.2020.05.059

Sosa, M., Zarco, R., y Postiglioni, A. (2006). Modelando aspectos de grupo en entornos colaborativos para proyectos de investigación. Revista de Informática Educativa y Medios Audiovisuales, 3(7), 22-31.

Tagle, D., Granados, G. y Rodríguez, J. (2020). Estrategias empresariales ante la COVID-19: el caso de la industria curtidora de León, Guanajuato. Entreciencias: Diálogos en la Sociedad del Conocimiento. DOI:10.22201/enesl.20078064e.2020.22.76844e22.76844

Thompson, A. \& Strickland, A. (2004). Administración estratégica. McGraw Hill.

Tobarra, M., Montero, F., \& Gallud, J. (2008). Usabilidad colaborativa: Caracterizando la usabilidad en entornos colaborativos. In IX Congreso Internacional Interacción, Albacete.

Uribe Beltrán, C. I., y Sabogal Neira, D. F. (2021). Marketing digital en micro y pequeñas empresas de publicidad de Bogotá. Revista Universidad \& Empresa, 23(40), 1-22. https://doi.org/10.12804/revistas.urosario.edu.co/empresa/a.8730

Van de Vrande, V., de Jong, J. P. J., Vanhaverbeke, W. y de Rochemont, M. (2009). Open innovation in SMEs: Trends, motives and management challenges. Technovation, 29, 423-437.

Viveros, N. L. H., y Camargo, F. F. (2020). Administración colaborativa: el nuevo desafío para el administrador de empresas, como líder en las organizaciones. Revista of business and entrepreneurial studies, 4(2). 
Volberda, H.W. (1996). Toward the flexible form: how to remain vital in hypercompetitive environments. Organization Science, 7(4), 359-374. https://doi.org/10.1002/smj.4250050302

Vrgovic, P., Vidicki, P., Glassman, B. y Walton, A. (2012). Open innovation for SMEs in developing countries - Anintermediated communication network model for collaboration beyond obstacles. Innovation: Management, Policy \& Practice, 14(3), 290-302.

Yoguel, G. y Boscherini, F. (1996). La capacidad de innovación y el fortalecimiento de la competitividad de las firmas: el caso de las PyMEs exportadoras argentinas. CEPAL, documento de trabajo No. 71. 